\title{
山东省济宁地区新生儿脂肪酸氧化代谢病笁查 及随访分析
}

杨池菊 ${ }^{1}$, 史彩虹 ${ }^{2}$, 周 成 ${ }^{1}$, 万秋花 ${ }^{1}$, 周艳彬 ${ }^{1}$, 陈西贵 ${ }^{1}$, 靳宪莲 ${ }^{1}$, 黄成刚 ${ }^{3}$, 徐 鹏 $^{1}$

1. 济宁市妇幼保健计划生育服务中心新生儿疾病篮查中心, 山东济宁 272000

2. 济宁市第二人民医院检验科, 山东济宁 272000

3. 浙江博圣生物技术股份有限公司, 浙江杭州 310012

[摘 要] 目的:了解山东省济宁地区脂肪酸氧化代谢病的发病率、基因突变特 征, 并评估治疗效果。方法: 采集 2014年7月14日-2019年12月31日出生的新生 儿血样, 用串联质谱法测定血肉碱和酰基肉碱水平, 篮查脂肪酸氧化代谢病。提取 篎查阳性新生儿外周血DNA, 用 MassARRAY 和高通量测序进行基因突变分析, 用 桑格一库森法验证。对确诊患儿早期干预治疗并随访。结果: 从608 818 名新生儿 中篎查出脂肪酸氧化代谢病患儿 42 例, 总发病率为 $1 / 14496$ 。以原发性肉碱缺乏 症 (16例,38.10\%)和短链酰基辅酶 A 脱氢酶缺乏症(16例,38.10\%)多见, 其次为极 长链酰基辅酶 $\mathrm{A}$ 脱氢酶缺乏症 (6例, 14.29\%) 和中链酰基辅酶 $\mathrm{A}$ 脱氢酶缺乏症 (4例, 9. 53\%)。原发性肉碱缺乏症患儿SLC22A5突变以c. 1400C > G (p. S467C) 和 c. $51 \mathrm{C}>\mathrm{G}($ p. F17L) 常见, 新发现 c. 278C >T (p. S93L)、c. 1049T >C (p. L350P)、 c. $572 A>G(p . K 191 R) 、$ c. $431 T>C(p . L 144 P)$ 突变。随访期内, 肉碱替代治疗 10 例 患儿发育正常; 未用肉碱替代治疗 6 例患儿中 5 例发育正常, 另 1 例新生儿期出现低 血糖, 肌酸激酶增高, 后期出现智力和语言发育落后。短链酰基辅酶 $\mathrm{A}$ 脱氢酶缺乏 症患儿ACADS基因突变以 c. 1031 A>G (p. E344G)和c. 164C > T (p. P55L)常见, 随访 期内发育正常。极长链酰基辅酶 $\mathrm{A}$ 脱氢酶缺乏症患儿 ACADVL基因突变以 c. 1349G >A (p. R450H) 常见, 新发现c. 488T >A(p. L163*)、c. 1228G >T(p. D410Y)、 c. $1276 \mathrm{G}>\mathrm{A}($ p. A426T)、c. $1522 \mathrm{C}>\mathrm{T}($ p. Q508*)、c. 1226C $>\mathrm{T}($ p. T409M) 突变。3例使 用中链脂肪酸奶粉患儿随访期内发育正常; 3 例合并肉碱降低患儿使用左卡尼汀和 中链脂肪酸奶粉治疗,其中 1 例患儿随访期内发育正常, 1 例患儿 3 月龄时急性发病 死亡, 1例患儿 8 月龄时曾急性发病, 治疗后症状消失, 随访期内发育正常。中链酰 基辅酶 A 脱氢酶缺乏症患儿ACADM 基因突变以 c. 449_452del(p. T150Rfs*4)常见， 新发现 c. 718A > G (p. M240V) 突变。所有患儿确诊后进行低脂肪饮食并避免饥饿和 疲劳, 1 例患儿补充左卡尼汀, 其余 3 例患儿未使用药物治疗, 随访期内发育均正

收稿日期:2021-04-26 接受日期:2021-08-04

基金项目: 济宁市重点研发计划(2020YXNS049)

第一作者: 杨池菊,副主任技师,主要从事新生儿遗传代谢病笁查及实验室诊断研究;E-mail :zyzza2@126.com; https://orcid. org/0000-0003-4628-1787

通信作者: 徐 鹏, 副主任医师,主要从事儿童内分泌和遗传代谢病诊断和治疗研究;E-mail:1365216874@qq.com; https ://orcid.org/0000-0002-1933-1219 
常。结论: 济宁地区脂肪酸氧化代谢病以原发性肉碱缺乏症和短链酰基辅酶A 脱氢 酶缺乏症常见, 存在基因热点突变或新发现的基因突变, 通过新生儿篮查早期诊 治, 患儿预后良好。

[关键词]脂质代谢缺陷, 先天性; 基因突变; 串联质谱法; 新生儿篮查; 随访研究 [中图分类号 ］ R596;R722.11;R446［文献标志码］ A

\section{Screening and follow-up results of fatty acid oxidative metabolism disorders in 608818 newborns in Jining, Shandong province}

YANG Chiju ${ }^{1}$, SHI Caihong ${ }^{2}$, ZHOU Cheng ${ }^{1}$, WAN Qiuhua ${ }^{1}$, ZHOU Yanbin ${ }^{1}$, CHEN Xigui $^{1}$, JIN Xianlian ${ }^{1}$, HUANG Chenggang ${ }^{3}$, XU Peng ${ }^{1}$ (1. Neonatal Disease Screening Center, Jining Maternal and Child Health and Family Planning Service Center, Jining 272000, Shandong Province, China; 2. Clinical Laboratory, Jining Second People's Hospital, Jining 272000, Shandong Province, China; 3. Zhejiang Bosheng Biotechnology Co., Ltd., Hangzhou 310012, China)

Corresponding author: XU Peng, E-mail: 1365216874@qq.com, https: //orcid.org/00000002-1933-1219

[ Abstract ] Objective: To investigate the incidence and gene mutation characteristics of fatty acid oxidative metabolism disorders in Jining area of Shandong province, and to evaluate the therapeutic effect. Methods: Blood samples of newborns were collected in Jining of Shandong province between July 14, 2014 and December 31, 2019. Tandem mass spectrometry was used to determine the levels of carnitine and acylcarnitine in the blood to screen for fatty acid oxidative metabolism disorder. For newborns with positive screening result, blood DNA was analyzed by MassARRAY and high-throughput sequencing, then verified by Sanger sequencing. The diagnosed children were given early intervention and treatment, and followed up. Results: Forty-two children with fatty acid oxidative metabolism disorders were screened out of 608818 newborns, with an incidence rate of 1/14 496. Primary carnitine deficiency (16 cases, 38.10\%) and shortchain acyl-CoA dehydrogenase deficiency (16 cases, 38.10\%) were the most common, followed by very long-chain acyl-CoA dehydrogenase deficiency (6 cases, 14.29\%), medium-chain acyl-CoA dehydrogenase deficiency (4 cases, 9.53\%). In children with primary carnitine deficiency, c.1400C $>\mathrm{G}$ (p.S467C) and c.51C $>\mathrm{G}$ (p.F17L) were the most common in SLC22A5 mutations; and c.278C >T (p.S93L), c.1049T >C (p.L350P), c.572A $>$ G (p.K191R), c.431T>C (p.L144P) were newly discovered mutations. Ten children with carnitine replacement therapy showed normal development during the follow-up. In 6 children without carnitine replacement treatment, hypoglycemia developed during the neonatal period in 1 case, in whom the creatine kinase was increased, and the intellectual and language development delayed in the later period; the other 5 children developed normally during the follow-up period. The ACADS gene mutations c.1031A $>\mathrm{G}$ (p.E344G) and c.164C>T (p.P55L) were common in children with short-chain acyl-CoA dehydrogenase deficiency, and the children developed normally during the follow-up. In 
children with very long-chain acyl-CoA dehydrogenase deficiency, the c.1349G>A (p.R450H) was common in ACADVL gene mutations; and c.488T >A (p.L163*), c.1228G>T (p.D410Y), c.1276G>A (p.A426T), c.1522C>T (p.Q508*), c.1226C >T (p.T409M) were newly discovered mutations. Three children treated with milk powder rich in mediumchain fatty acids had normal development during the follow-up. The other 3 cases with combined carnitine reduction were treated with levocarnitine and milk powder enriched of medium-chain fatty acids, 1 case developed normally during the follow-up, 1 case died of acute illness at the age of 3 months, and 1 case had acute illness and recovered after treatment, and developed normally during the follow-up. c.449_452del (p.T150Rfs*4) was the most common $A C A D M$ gene mutation in children with medium-chain acyl-CoA dehydrogenase deficiency, and c. 718A >G (p.M240V) was a newly discovered mutation. All children received low-fat diet, and hunger and fatigue were avoided; 1 child was supplemented with L-carnitine, and the other 3 children were not treated with drugs, and all of them developed normal during the follow-up. Conclusions: Primary carnitine deficiency and short-chain acyl-CoA dehydrogenase deficiency are the most common fatty acid oxidative metabolism disorders in Jining area. There are gene hotspot mutations and new discovered gene mutations in patients. Patients with early diagnosis and treatment through neonatal screening have a good prognosis.

[ Key words ] Lipid metabolism, inborn errors; Gene mutation; Tandem mass spectrometry; Neonatal screening; Follow-up studies

[J Zhejiang Univ (Med Sci), 2021, 50(4): 472-480.]

[缩略语] 游离肉碱( free carnitine, C0); 乙酰肉碱(acetylcarnitine, C2); 丙酰肉碱 (propionylcarnitine, C3); 丁酰肉碱(butyrylcarnitine, C4); 辛酰肉碱(octanoylcarnitine, C8); 葵酰肉碱 (decanoylcarnitine, C10); 肉豆冦烯酰肉碱 (tetradecenoylcarnitine, C14:1); 棕桐酰肉碱 (palmitoylcarnitine, C16); 聚合酶链反应 (polymerase chain reaction, PCR)

脂肪酸氧化代谢病是一种常见的新生儿遗 传代谢病, 是由于脂肪酸进人线粒体进行 $\beta$ 氧化 代谢途径中的酶或转运蛋白功能缺陷, 导致脂肪 酸 $\beta$ 氧化代谢发生障碍所引起的一组疾病; 属于 常染色体隐性遗传病, 发病时多累及肝脏、心肌 和骨骼肌, 导致患儿运动发育落后、肌无力、肝 大、低血糖、酸中毒甚至猝死。串联质谱法用于 新生儿篮查可以实现脂肪酸氧化代谢病的早期 诊断和及时治疗。本文回顾性分析了山东省济 宁市 2014-2019年新生儿遗传代谢病串联质谱 法篎查资料, 分析脂肪酸氧化代谢病篮查确诊患 儿的基因突变特征和治疗效果, 探讨脂肪酸氧化 代谢病患儿的基因型与临床表型、治疗效果的关 系, 为脂肪酸氧化代谢病的精准诊断和早期干预
提供参考。

\section{1 对象与方法}

\section{1 对 象}

篮查对象为山东省济宁地区 2014 年 7 月 14 日-2019年 12 月 31 日出生的新生儿共608 818 名, 其中男性335558名, 女性273260名, 男女比 例为 $1.23: 1$; 正常足月儿596249名, 早产儿 4023 名,过期产儿8546名; 正常体重儿596215名, 低出生体重儿3972名,巨大儿8631名。健康新生 儿出生后 3 7 d充分哺乳后采血, 早产儿和低出生 体重儿采血时间不超过出生后 $20 \mathrm{~d}$ 。本研究中的 标本采集和检测经监护人知情同意,且研究方案 通过济宁市妇幼保健计划生育服务中心伦理委员 
会批准。

\section{2 仪器及试剂}

S\&S903采血滤纸为英国 Whatman 公司产品; AQCUTY TQ-D 三重四极杆串联质谱仪为美国 Waters 公司产品; MassARRAY Analyzer 4核酸质谱 仪和 RS-1000芯片点样机为美国 Sequeno 公司产 品;Illumina HiSeq 2500 测序平台为美国 Illumina 公司产品; ABI 3500XL基因分析仪为美国 Applied Biosystems 公司产品。

NeoBase 新生儿非衍生化法筛查试剂盒为芬 兰PerkinElmer 公司产品; DNA 提取试剂盒为德国 Qiangen公司产品; 核酸质谱分析 MassARRAY 试剂 盒为美国 Agena公司产品; 高通量测序 Invitrogen Qubit dsDNA 检测试剂盒为美国 Invitrogen 公司产 品; Covaris LE220 试剂盒为美国 Covaris 公司产品; Illumina DNA 标准和引物预混试剂盒为美国 Kapa Biosystems 公司产品; 桑格-库森法 PCR 试剂盒为日 本 TaKaRa公司产品; PCR 产物纯化试剂盒为德国 Macherey-Nagel 公司产品;BigDye Terminatorv 3. 1 循环测序试剂盒为美国Applied Biosystems 公司 产品。

\section{3 串联质谱法笁查脂肪酸氧化代谢病}

采集新生儿足跟末梢血滴于采血滤纸上,采 用非衍生化串联质谱法测定干血斑 $\mathrm{C} 0$ 和酰基肉 碱含量。串联质谱法检测的 $\mathrm{C} 0$ 和酰基肉碱正常 参考区间采用百分位数法, 并通过模型分析确定 各自疾病的特异性篮查指标。其中, 原发性肉碱 缺乏症为 C0 降低 (正常参考值 $10 \sim 55 \mu \mathrm{mol} / \mathrm{L}$ ), 可 伴多种酰基肉碱降低判定篮查阳性; 短链酰基辅 酶 $\mathrm{A}$ 脱氢酶缺乏症为 $\mathrm{C} 4$ 升高 (正常参考值 0.07 $0.45 \mu \mathrm{mol} / \mathrm{L}$ ), 可伴 $\mathrm{C} 4 / \mathrm{C} 3$ 比值 (正常参考值 0. 05 0. 42) 升高判定篮查阳性; 极长链酰基辅酶 $\mathrm{A}$ 脱氢酶缺乏症为 $\mathrm{C} 14: 1$ 升高 (正常参考值 0.02 $0.22 \mu \mathrm{mol} / \mathrm{L}$ ), 可伴 $\mathrm{C} 14: 1 / \mathrm{C} 2$ 比值 (正常参考值 0 0.01 ) 或 C14:1/C16 比值 (正常参考值 0 0.09) 升 高判定篮查阳性; 中链酰基辅酶 $\mathrm{A}$ 脱氢酶缺乏症 为 $\mathrm{C} 8$ 升高 (正常参考值 $0.01 \sim 0.12 \mu \mathrm{mol} / \mathrm{L}$ ), 可伴 $\mathrm{C} 8 / \mathrm{C} 2$ 比值 (正常参考值 $0 \sim 0.01$ ) 或 $\mathrm{C} 8 / \mathrm{C} 10$ 比值 (正常参考值 $0.40 \sim 1.33$ ) 升高判定篮查阳性 ${ }^{[1-2]}$ 。

1.4 核酸质谱法、高通量测序、桑格-库森法检 测基因突变

節查阳性新生儿召回复查篮查指标仍为阳性 者,采集其外周血, 提取 DNA。一部分患儿先用核
酸质谱法检测已知基因突变,若未发现基因突变, 再用高通量测序检测; 另一部分患儿直接用高通 量测序检测基因突变。阳性结果用桑格-库森法 验证。

1.4.1 核酸质谱法 采用核酸质谱分析系统 检测脂肪酸氧化代谢病 4 种常见基因 (ACADS、 $A C A D M 、 A C A D V L$ 和 $S L C 22 A 5)$ 的突变情况。按照试 剂盒说明,应用多重PCR 扩增目标靶序列, 然后使 用虾碱性磷酸酶移除PCR 反应残留的脱氧核苷三 磷酸, 使用延伸引物和测定特异性复合物终止子 核苷酸混合物,将单碱基延伸至突变位点,在干扰 离子与树脂结合后将产物转移到芯片上。使用核 酸质谱仪从飞行时间质谱图上检索质谱图, 用 SpectroTYPER 软件进行基因分型。

1.4.2 高通量测序 用紫外分光光度计检测 DNA 的浓度和纯度, 并将DNA 片段剪切至 150 $200 \mathrm{bp}$ 。通过多重PCR 将剪切的DNA用于靶区域 的文库制备, 用DNA标准和引物预混试剂盒进行 定量, HiSeq 2500 平台测序后, 与人类基因组参考 序列进行比对,使用 GATK软件对突变进行命名, 用ANNOVAR 软件对突变进行分类。参照美国医 学遗传学和基因组学学会联合分子病理协会提出 的 “序列变异解读标准和指南” 分析突变的致 病性 ${ }^{[3]}$ 。

1.4 .3 桑格-库森法 使用待测位点的特异性引 物, 按照试剂盒操作流程进行 PCR,PCR 产物纯化 后, 用 ABI 3500XL平台测序。测序结果采用 SeqMan 软件进行序列比对分析。根据脂肪酸氧化 代谢病的基因检测结果, 与 PubMed 数据库、dbSNP 数据库、千人基因组 (1000 Genomes) 数据库、人类 基因突变数据库等收录的基因突变信息比较,确 定脂肪酸氧化代谢病的基因热点突变或新发现的 基因突变。

\section{5 诊断标准}

满足下面其中一项即诊断为脂肪酸氧化代谢 病: (1)串联质谱法篎查特征性指标阳性, 且检测到 相应的基因复合杂合突变、纯合突变; (2如果检测 到杂合突变,特征性指标随访持续明显异常,或者 出现相应的临床症状 ${ }^{[4]}$ 。

\section{6 治疗和随访}

对于脂肪酸氧化代谢病确诊病例, 嘱其避免 饥饿和剧烈运动。原发性肉碱缺乏症患儿使用肉 碱替代治疗,按照50 100 mg $\mathrm{kg}^{-1} \cdot \mathrm{d}^{-1}$ 剂量补充 
左卡尼汀, 维持血 $\mathrm{CO}$ 正常或接近正常水平; 短链 酰基辅酶 $\mathrm{A}$ 脱氢酶缺乏症和中链酰基辅酶 $\mathrm{A}$ 脱氢 酶缺乏症患儿若出现血 C0 降低可适当补充左卡 尼汀; 极长链酰基辅酶 $\mathrm{A}$ 脱氢酶缺乏症患儿以高 糖低脂饮食治疗为主, 尤其要控制长链脂肪酸摄 人, 使用富含中链脂肪酸的奶粉, $\mathrm{C} 0$ 降低的患儿补 充左卡尼汀。急性期治疗主要为补充足量葡萄 糖、纠正酸中毒、电解质紊乱和对症治疗。

患儿病情稳定后 2 3个月随访复查一次, 随 访内容主要包括体格检查、智力发育、血肉碱水 平、肝功能、心肌酶谱、心电图及心脏彩超检查等, 随访时间截至2020年12月 31 日。

\section{7 统计学方法}

采用SPSS 20.0 软件进行统计分析, 正态分布 的计量资料用均数 \pm 标准差表示, 非正态分布的 计量资料用中位数 (范围) 表示; 计数资料采用例 数(百分率)表示。

\section{2 结 果}

确诊 4 种脂肪酸氧化代谢病 42 例, 发病率为 1/14 496。其中原发性肉碱缺乏症 16 例(38.10\%), 发病率为 $1 / 38051$; 短链酰基辅酶 $\mathrm{A}$ 脱氢酶缺乏症 16 例 (38.10\%), 发病率为 $1 / 38051$; 极长链酰基 辅酶 $\mathrm{A}$ 脱氢酶缺乏症 6 例 (14.29\%), 发病率为 $1 / 101470$; 中链酰基辅酶 $\mathrm{A}$ 脱氢酶缺乏症 4 例 $(9.53 \%)$, 发病率为 $1 / 152205$ 。

2.116 例原发性肉碱缺乏症患儿检测和随访 结果

16 例原发性肉碱缺乏症患儿中, 男性 8 例, 女 性 8 例; 早产儿 1 例, 足月儿 15例; 确诊时均无临床 症状, 确诊时间为 $45(21 \sim 57)$ 日龄。检出SLC22A5 基因突变 11 种, 其中纯合突变3例(18.75\%), 复合 杂合突变 11 例 $(68.75 \%$ ), 杂合突变 2 例 $(12.50 \%) 。$ 突变频率较高的位点为 c. $1400 \mathrm{C}>\mathrm{G}(\mathrm{p} . \mathrm{S} 467 \mathrm{C})$ $(14 / 30,46.67 \%)$ 和 c. $51 \mathrm{C}>\mathrm{G}$ (p. F17L) $(6 / 30$, $20.0 \%)$, 突变频率较低的位点包括 c. $95 \mathrm{~A}>\mathrm{G}$ (p. N32S )、c. 428C >T (p. P143L)、c. 246C >T (p. R82R)、c. 505C $>\mathrm{T}($ p. R 169W )、c. 278C $>\mathrm{T}$ (p. S93L) 、c. 1049T $>$ C (p. L350P )、c. 572A $>$ G (p. K191R)、c. 431T $>$ C (p. L144P) 和 c. 695C $>$ T (p.T232M)。新发现 c. 278C > T (p.S93L)、 c. $1049 \mathrm{~T}>\mathrm{C}($ p. L350P $) 、$ c. 572A>G (p. K191R)、 c. 431T $>\mathrm{C}(p . \mathrm{L} 144 \mathrm{P})$ 突变位点。
16 例患儿串联质谱法篮查 $\mathrm{C} 0$ 浓度为 5.90 (1.85 8.91) $\mu \mathrm{mol} / \mathrm{L}$,均低于正常范围。治疗随访 35 (10 69) 个月, 其中 9例患儿长期补充左卡尼 汀，血 $\mathrm{C} 0$ 浓度为 $(11.54 \pm 2.44) \sim(32.68 \pm$ 3. 36) $\mu \mathrm{mol} / \mathrm{L}$, 均在正常范围, 智力和语言发育正 常; 1 例患儿补充左卡尼汀 4 年后自行停药, 血 CO (9.69 \pm 1.19$) \mu \mathrm{mol} / \mathrm{L}$, 体格和智力发育正常。6例 未补充左卡尼汀患儿中 5 例患儿随访期内血 CO 浓 度为 $(4.32 \pm 0.62) \sim(7.63 \pm 1.33) \mu \mathrm{mol} / \mathrm{L}$, 仍低 于正常范围,但体格和智力发育正常; 1 例患儿新 生儿期出现低血糖, 肌酸激酶增高 $(362 \mathrm{U} / \mathrm{L})$, 随 访 $\mathrm{C} 0$ 为 $(4.32 \pm 0.62) \mu \mathrm{mol} / \mathrm{L}$, 随后出现智力和语 言发育落后。

结果提示, 原发性肉碱缺乏症为济宁地区较 为常见的脂肪酸氧化代谢病之一, c. $1400 \mathrm{C}>\mathrm{G}$ (p. S467C) 和 c. $51 \mathrm{C}>\mathrm{G}$ (p. F17L) 为热点突变, 早期 使用肉碱替代治疗患儿预后较好。

\subsection{6 例短链酰基辅酶 $\mathrm{A}$ 脱氢酶缺乏症患儿检} 测和随访结果

短链酰基辅酶 A 脱氢酶缺乏症患儿中,男性 8 例, 女性 8 例, 均为足月儿, 确诊时体格检查未见 异常,无临床症状, 确诊时间为 43(29 57) 日龄。 检出 $A C A D S$ 基因突变 7 种, 其中纯合突变 3 例 $(18.75 \%)$, 复合杂合突变 10 例 $(62.50 \%)$, 杂合突变 3 例 $(18.75 \%)$ 。突变频率较高的位点为 c. $1031 \mathrm{~A}>$ G (p. E344G) $(12 / 29,41.38 \%)$ 和c. 164C>T(p. P55L) $(9 / 29,31.03 \%)$, 突变频率较低的位点包括 c. $989 \mathrm{G}>\mathrm{A}($ p. R330H) 、c. 682_683del(p. E228Rfs*16)、 c. $1054 \mathrm{G}>\mathrm{A}($ p. A352T) 、c. $1130 \mathrm{C}>\mathrm{T}($ p. P337L $)$ 和 c. $682 \mathrm{G}>\mathrm{A}(\mathrm{p} . \mathrm{E} 228 \mathrm{~K}) 。$

16 例患儿串联质谱法初篎 $\mathrm{C} 4$ 浓度为 1.58 $(0.65 \sim 2.73) \mu \mathrm{mol} / \mathrm{L}, \mathrm{C} 4 / \mathrm{C} 3$ 比值为 $1.40(0.81 \sim$ 4.13)。治疗随访中位时间 43(13 73) 个月,C4浓 度为 $(0.60 \pm 0.02) \sim(2.23 \pm 0.13) \mu \mathrm{mol} / \mathrm{L}, \mathrm{C} 4 / \mathrm{C} 3$ 比值为 $(0.69 \pm 0.02) \sim(3.29 \pm 0.39)$, 体格和智力 发育均正常。

结果提示, 短链酰基辅酶 $\mathrm{A}$ 脱氢酶缺乏症为 济宁地区较为常见的脂肪酸氧化代谢病之一, c. $1031 \mathrm{~A}>\mathrm{G}$ (p. E344G) 和c. 164C>T(p. P55L)为热 点突变,早期饮食干预后患儿预后较好。

\subsection{6 例极长链酰基辅酶 $\mathrm{A}$ 脱氢酶缺乏症患儿} 检测和随访结果

6 例极长链酰基辅酶 $\mathrm{A}$ 脱氢酶缺乏症患儿均 
为男性、足月儿, 确诊时体格检查未见异常, 无临 床症状, 确诊时间为 44(35 50) 日龄。检出 $A C A D V L$ 基因突变 9 种, 突变频率较高的位点为 c. $1349 \mathrm{G}>\mathrm{A}(\mathrm{p} . \mathrm{R} 450 \mathrm{H})(3 / 11,27.27 \%)$, 突变频率 较低的位点为 c. $1280 \mathrm{G}>\mathrm{A}(\mathrm{p} . \mathrm{W} 427 *) 、$ c. $1328 \mathrm{~T}>\mathrm{C}$ (p. M443T)、c. 996dup (p. A333Cf*26)、c. 488T >A (p. L163*)、c. 1228G >T(p. D410Y)、c. 1276G >A (p. A426T)、c. 1522C >T (p. Q508*)和 c. 1226C $>\mathrm{T}$ (p. T409M)。新发现 c. 488T>A (p. L 163*)、 c. $1228 \mathrm{G}>\mathrm{T}$ (p. D410Y)、c. 1276G $>\mathrm{A}($ p. A426T)、 c. $1522 \mathrm{C}>\mathrm{T}($ p. Q508*)、c. 1226C $>\mathrm{T}($ p. T409M) 突 变位点。

6 例患儿串联质谱法初篮 C14:1 浓度为 1.63 (0. 67 4. 65) $\mu \mathrm{mol} / \mathrm{L}, \mathrm{C} 14: 1 / \mathrm{C} 2$ 和 C14:1/C16 比值 分别为 $0.22(0.04 \sim 0.67)$ 和 $0.46(0.31 \sim 1.00)$, 均 高于正常范围。治疗随访中位时间 20(3 49) 个 月, 3 例 $\mathrm{C} 0$ 正常患儿采用中链脂肪酸奶粉治疗, C14:1、C14:1/C2 和 C14:1/C16 比值分别为 $(0.20 \pm$ $0.01) \sim(2.61 \pm 0.32) \mu \mathrm{mol} / \mathrm{L} 、(0.01 \pm 0.01) \sim$ $(0.32 \pm 0.06)$ 和 $(0.07 \pm 0.01) \sim(0.91 \pm 0.10)$, 均 高于正常范围, 体格和智力发育正常; 3 例合并 $\mathrm{C} 0$ 降低的患儿使用左卡尼汀和中链脂肪酸奶粉治 疗, 其中 1 例 c. 488T $>A(p . L 163 *)$ 杂合突变患儿 3 月龄时因肺炎急性发病死亡; 1 例患儿 8 月龄时 急性发病, 出现心力衰竭、心源性肺水肿、肥厚性 心肌病,急性期增加服用地高辛、贝那普利及利尿 药物治疗后症状缓解; 1 例患儿随访期内无急性发 病,体格和智力发育正常。

结果提示, 极长链酰基辅酶 $\mathrm{A}$ 脱氢酶缺乏症 在济宁地区发病率较低,c. 1349G >A (p. R450H) 为 热点突变, 临床危害较重, 治疗期间仍有部分病例 死亡或急性发病。

2.44 例中链酰基辅酶 A 脱氢酶缺乏症患儿检 测和随访结果

4 例中链酰基辅酶 $\mathrm{A}$ 脱氢酶缺乏症患儿中男 性 2 例, 女性 2 例, 均为足月儿, 确诊时体格检查正 常, 无临床症状, 确诊时间为 $(51 \pm 6)$ 日龄。检出 $A C A D M$ 基因突变 4 种, 突变频率较高的位点为 c. 449_452del(p. T150Rfs*4) $(3 / 6,50.0 \%)$, 突变 频率较低的位点包括 c. $718 \mathrm{~A}>\mathrm{G}$ (p. M240V)、 c. $928 \mathrm{G}>\mathrm{A}($ p. G310R) 和 c. $157 \mathrm{C}>\mathrm{T}$ (p. R53C), 新发 现 c. $718 \mathrm{~A}>\mathrm{G}(\mathrm{p} . \mathrm{M} 240 \mathrm{~V})$ 突变位点。4例患儿 $\mathrm{C} 8$ 浓度为 $4.56(0.62 \sim 11.29) \mu \mathrm{mol} / \mathrm{L}, \mathrm{C} 8 / \mathrm{C} 2$ 和 C8/
C 10 比值分别为 $0.45(0.04 \sim 0.67)$ 和 9.97 (2. 69 16.71), 均高于正常范围。确诊后所有患 儿采用低脂肪饮食, 避免饥饿和疲劳, 随访中位时 间 36(13 54) 个月, 1 例合并 C0 降低的患儿服用左 卡尼汀治疗, 其余 3 例患儿未用药物治疗, 随访 $\mathrm{C} 8 、 \mathrm{C} 8 / \mathrm{C} 2 、 \mathrm{C} 8 / \mathrm{C} 10$ 分别为 $(0.59 \pm 0.08) \sim(2.11 \pm$ $0.23) \mu \mathrm{mol} / \mathrm{L} 、(0.04 \pm 0.01) \sim(0.65 \pm 0.13)$ 、 (2. $82 \pm 0.35) \sim(11.32 \pm 2.50)$, 随访期内患儿体 格和智力发育均正常。结果提示, 中链酰基辅 酶 $\mathrm{A}$ 脱氢酶缺乏症在济宁地区发病率较低, c. 449_452del(p. T150Rfs*4)为热点突变, 早期低 脂肪饮食干预患儿预后较好。

\section{3 讨 论}

脂肪酸氧化代谢病的发病率在不同地区和人 种间差异很大,其在美国、澳大利亚、德国、意大利 等国家发病率相对较高 ${ }^{[5-9]}$, 在亚洲国家中并不常 见 $^{[10-11]}$ 。Chace 等 $^{[12]}$ 对美国宾夕法尼亚州、北卡 罗来纳州和华盛顿特区及俄亥俄州东部地区 710000 名新生儿篮查结果显示, 脂肪酸氧化代谢 病的发病率为 $1 / 15000$; Schulze 等 ${ }^{[13]}$ 报道德国 250000 名新生儿篎查结果显示, 脂肪酸氧化代谢 病发病率为 $1 / 10400$; 脂肪酸氧化代谢病在日本 的发病率为 $1 / 30000$, 在韩国的发病率为 $1 /$

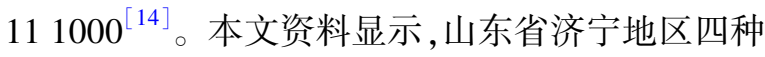
脂肪酸氧化代谢病的发病率为 $1 / 14496$, 与上海市 和浙江省的研究结果接近 ${ }^{[15-16]}$ 。其中, 原发性肉 碱缺乏症和短链酰基辅酶 $\mathrm{A}$ 脱氢酶缺乏症为济宁 地区最常见的两种脂肪酸氧化代谢病, 各占 $38.10 \%$, 其他疾病所占比例较小。

本文资料中, 原发性肉碱缺乏症检出率达到 $1 / 38051$ 。血 C0 水平和基因检测是篮查原发性肉 碱缺乏症的主要依据 ${ }^{[17]}$ 。本文资料中的 16 例患 儿均出现 C0 降低, 其中检测到SLC22A5纯合和复 合杂合突变 14 例, 杂合突变 2 例, 其中 2 例杂合突 变患儿初篮时血 CO 降低、用药后恢复正常、停药 后再次明显降低而确诊。因此, 在检出一个基因 位点突变时, 应反复检测血 $\mathrm{C} 0$ 水平, 若血 $\mathrm{C} 0$ 水平 持续明显异常, 应诊断为阳性病例, 若停药后 $\mathrm{C} 0$ 水平降低不明显,应检测患儿母亲血 $\mathrm{C} 0$ 水平和基 因突变,以排除母源性肉碱缺乏。SLC22A5基因突 变类型多为错义突变,其次为无义突变及移码突 变 ${ }^{[18]}$ 。不同种族人群的基因突变谱有一定的差 
异 ${ }^{[19]}$, 某些种族和地区人群中存在热点突变, 如 高加索人常见的突变位点为 c. $632 \mathrm{~A}>\mathrm{G}$, 意大利常 见的突变位点为 c. $505 \mathrm{C}>\mathrm{T}$, 西非人群以 c. $632 \mathrm{~A}>\mathrm{G}$ 为主, 日本以 c. 1400C $>\mathrm{G}$ 和 c. 396G $>A$ 突变最常 见 $^{[20]}$ 。Lee 等 ${ }^{[18]}$ 报道中国台湾地区以 c. 760C $>\mathrm{T}$ (p. R254X) 最常见。崔冬等 ${ }^{[21]}$ 报道了8 例原发性 肉碱缺乏症患者, 6 例检出突变位点 c. $760 \mathrm{C}>\mathrm{T}(\mathrm{p}$. $\mathrm{R} 254 \mathrm{X}$ )。本文资料 16 例患儿检出频率最高的突 变位点为 c. $1400 \mathrm{C}>\mathrm{G}(\mathrm{p} . \mathrm{S} 467 \mathrm{C})$, 其次为 c. $51 \mathrm{C}>\mathrm{G}$ (p. F17L), 与天津地区报道的结果基本一致 ${ }^{[22]}$ 。 原发性肉碱缺乏症可在任何年龄发病, 若不能及 时治疗, 患者有猝死的风险, 早期使用肉碱替代治 疗是改善患者预后的关键 ${ }^{[23]}$ 。本文资料中, 1 例 患儿在新生儿期出现血糖降低, 肌酸激酶增高, 但 家长拒绝补充左卡尼汀, 随访期内出现智力和语 言发育落后, 该患儿存在济宁地区新发现突变位 点 c. 431T $>C$ (p. L144P) 与 c. 695C $>T$ (p. T232M) 的 复合杂合突变。c. $1400 \mathrm{C}>\mathrm{G}($ p. $S 467 \mathrm{C})$ 热点突变 患儿预后较好, 可能与该基因是原发性肉碱缺乏 症轻微临床表型突变基因有关 ${ }^{[24]}$,也与新生儿篮 查后早期干预有关。

C4 浓度增加是短链酰基辅酶 $\mathrm{A}$ 脱氢酶缺乏 症诊断的重要标志物 ${ }^{[25-26]}$ 。本文资料中的 16 例 短链酰基辅酶 $\mathrm{A}$ 脱氢酶缺乏症患儿 $\mathrm{C} 4 、 \mathrm{C} 4 / \mathrm{C} 2$ 和 $\mathrm{C} 4 / \mathrm{C} 3$ 均异常。ACADS突变在欧洲以 c. $511 \mathrm{C}>\mathrm{T}$ 和 c. $625 \mathrm{G}>\mathrm{A}$ 突变最常见, 美国 c. $511 \mathrm{C}>\mathrm{T}$ 的等位基 因频率为 $0.3 \%, \mathrm{c} .625 \mathrm{G}>\mathrm{A}$ 的等位基因频率为 $5.5 \%$ [27-28]。常见突变是错义突变, 通过改变折叠 方式和细胞的寿命来影响蛋白质的生物合 成 ${ }^{[29]}$ 。Pedersen 等 ${ }^{[30]}$ 报道了 114 例短链酰基辅酶 A 脱氢酶缺乏症患者, c. $625 \mathrm{G}>\mathrm{A}$ ( p. G209S)和 c. $511 \mathrm{C}$ (p. R147W) 突变分别占所检测等位基因的 $67 \%$ 和 $8 \%$ 。本文资料中, 16 例患儿共检测到 8 种突变, 检出频率最高的突变为 c. $1031 \mathrm{~A}>\mathrm{G}$ (p. E 344G) 和 c. 164 C > T (p. P 5 5 L), 其次为 c. $682 \_683 \mathrm{del}($ p. E228Rfs*16) 和 c. $989 \mathrm{G}>\mathrm{A}$ (p. R330H), 与山东省青岛地区报道的结果接 近 $^{[31]}$, 提示 c. $1031 \mathrm{~A}>\mathrm{G}($ p. E344G) 突变可能是山 东地区的热点突变。大多数新生儿篮查确诊的短 链酰基辅酶 $\mathrm{A}$ 脱氢酶缺乏症患儿无明显症状, 但 也有出现喂养困难及低血糖等 ${ }^{[32]}$ 。本文资料中 的 16 例确诊患儿均无临床表现, 体格和智力发育 正常, 可能是由于新生儿篮查确诊的患儿多为良
性表现的缘故,也与确诊后及时进行生活管理和 喂养指导, 避免了临床症状出现及应急状态下的 代谢失调有关。

极长链酰基辅酶 $\mathrm{A}$ 脱氢酶缺乏症临床表现有 明显的异质性, 多为新生儿和肾儿早期发病, 常伴 有心肌受累, 猝死率较高。在本文资料 6 例极长链 酰基辅酶 $\mathrm{A}$ 脱氢酶缺乏症患儿中, 1 例患儿早期死 亡, 1 例患儿治疗期内急性发病。目前, $A C A D V L$ 以 错义突变为主要突变类型, 其次为缺失突变和剪 切突变 ${ }^{[33]}$ 。本文资料中的 6 例患儿有 4 例为 c. $1349 \mathrm{G}>\mathrm{A}$ (p. R450H) 突变, 提示该位点有较高的 发生频率, 为济宁地区患儿的热点突变。通过新生 儿篮查确诊的患儿尽管在诊断时多无临床症状,但 其发病和预后将出现较大差异, 有些患儿甚至在诊 断结果出来之前就已经有症状, 有些患儿长期随访 仍无症状 ${ }^{[34-35]}$ 。部分患儿会出现横纹肌溶解综合 征或严重的心肌病,这些严重的表型与等位基因失 活或无效有关 ${ }^{[36-37]}$, 因此评估患儿发病风险时,基 因检测显得尤为重要。本文资料中的 1 例死亡患 儿检出 c. 488T $>$ A (p. L163*)位点的杂合突变, 该突 变是本地区新发现的突变, 是否存在致病性尚须进 一步论证; 而3例携带c. 1349G >A (p. R450H)复合 杂合子的患儿虽然 C 1 4: 1 明显增高, 使用中链 脂肪酸奶粉治疗, 无急性发病, 发育正常, 推测 c. 1349G >A(p. R450H) 突变可能与血 C14:1生化表 型存在关联,而与临床表型关联不明显。

中链酰基辅酶 $\mathrm{A}$ 脱氢酶缺乏症是澳大利亚、 欧美等地区最主要的脂肪酸氧化代谢病,占 $50 \%$ 以上, 发病率为 $1 / 45000 \sim 1 / 9036^{[38-40]}$ 。中链酰基 辅酶A 脱氢酶缺乏症在我国发病率不高, 本文资料 显示济宁地区的发病率约为 $1 / 152$ 205。ACADM 以错义突变为主要类型, 欧美白种人最常见的突 变是位于第 11 外显子的 c. $985 \mathrm{~A}>\mathrm{G}(\mathrm{K} 329 \mathrm{E})^{[41]}$, 本 文资料未检出该突变位点。该病多为良性疾病, 发病多存在诱发因素, 如长时间饥饿或感染性疾 病等。患儿确诊后应避免饥饿, 提高饮食中碳水 化合物和蛋白质占比, 减少脂肪摄人, 以预防发 病。本文资料中确诊的 4 例中链酰基辅酶 $\mathrm{A}$ 脱氢 酶缺乏症患儿随访期内体格和智力发育正常。

综上所述, 济宁地区脂肪酸氧化代谢病以原 发性肉碱缺乏症和短链酰基辅酶 $\mathrm{A}$ 脱氢酶缺乏症 常见, 极长链酰基辅酶 $\mathrm{A}$ 脱氢酶缺乏症的临床表 型相对严重; 通过新生儿篮查早期发现、及时干 
预、规范治疗, 可以在一定程度上预防发病或减轻 疾病的危害。

\section{利益冲突 所有作者均声明不存在利益冲突}

\section{参考文献}

[1] MCHUGH D M S, CAMERON C A, ABDENUR J E, et al. Clinical validation of cutoff target ranges in newborn screening of metabolic disorders by tandem mass spectrometry: a worldwide collaborative $\operatorname{project}[\mathrm{J}]$. Genet Med, 2011, 13(3): 230-254.

[2] 马志军, 韩连书, 李水军, 等. MS/MS 技术在新生儿氨 基酸、有机酸及脂肪酸氧化代谢障碍性疾病篎查中 的应用共识 $[\mathrm{J}]$. 检验医学, 2019, 34(6): 479-485.

MA Zhijun, HAN Lianshu, LI Shuijun, et al. Expert consensus on neonatal disease screening of amino acid, organic acid and fatty acid oxidative metabolic disorders using MS/MS technology[J]. Laboratory Medicine, 2019, 34(6): 479-485. (in Chinese)

[3] RICHARDS S, AZIZ N, BALE S, et al. Standards and guidelines for the interpretation of sequence variants: a joint consensus recommendation of the American College of Medical Genetics and Genomics and the Association for Molecular Pathology $[\mathrm{J}]$. Genet Med, 2015, 17(5): 405-424.

[4] FRAZIER D M, MILLINGTON D S, MCCANDLESS S $\mathrm{E}$, et al. The tandem mass spectrometry newborn screening experience in North Carolina: 1997-2005[J]. J Inherit Metab Dis, 2006, 29(1): 76-85.

[5] WILCKEN B, WILEY V, HAMMOND J, et al. Screening newborns for inborn errors of metabolism by tandem mass spectrometry[J]. N Engl J Med, 2003, 348(23): 2304-2312.

[6] FEUCHTBAUM L, CARTER J, DOWRAY S, et al. Birth prevalence of disorders detectable through newborn screening by race/ethnicity $[\mathrm{J}]$. Genet Med, 2012, 14(11): 937-945.

[7] LINDNER M, GRAMER G, HAEGE G, et al. Efficacy and outcome of expanded newborn screening for metabolic diseases - report of 10 years from South-West Germany $[\mathrm{J}]$. Orphanet J Rare Dis, 2011, 6(1): 44.

[8] LA MARCA G, MALVAGIA S, CASETTA B, et al. Progress in expanded newborn screening for metabolic conditions by LC-MS/MS in Tuscany: update on methods to reduce false tests $[\mathbf{J}]$. J Inherit Metab Dis, 2008, 31(S2): 395-404.

[9] LIM J S, TAN E S, JOHN C M, et al. Inborn error of metabolism (IEM) screening in Singapore by electrospray ionization-tandem mass spectrometry (ESI/MS/ MS): an 8 year journey from pilot to current program $[\mathrm{J}]$. Mol Genet Metab, 2014, 113(1-2): 53-61.

[10] VILARINHO L, ROCHA H, SOUSA C, et al. Four years of expanded newborn screening in Portugal with tandem mass spectrometry $[\mathrm{J}]$. J Inherit Metab Dis, 2010, 33(S3): 133-138.

[11] NAGARAJA D, MAMATHA S N, DE T, et al. Screening for inborn errors of metabolism using automated electrospray tandem mass spectrometry: Study in high-risk Indian population $[\mathrm{J}]$. Clin Biochem, 2010, 43(6): 581-588.

[12] CHACE D H, DIPERNA J C, NAYLOR E W. Laboratory integration and utilization of tandem mass spectrometry in neonatal screening: a model for clinical mass spectrometry in the next millennium $[\mathrm{J}]$. Acta Paediatrica, 1999, 88(432): 45-47.

[13 ] SCHULZE A, LINDNER M, KOHLMÜLLER D, et al. Expanded newborn screening for inborn errors of metabolism by electrospray ionization-tandem mass spectrometry: results, outcome, and implications $[\mathrm{J}]$. Pediatrics, 2003, 111(6): 1399-1406.

[14] SHIBATA N, HASEGAWA Y, YAMADA K, et al. Diversity in the incidence and spectrum of organic acidemias, fatty acid oxidation disorders, and amino acid disorders in Asian countries: selective screening vs. expanded newborn screening $[\mathrm{J}]$. Mol Genet Metab Rep, 2018, 16: 5-10.

[15] HAN L, HAN F, YE J, et al. Spectrum analysis of common inherited metabolic diseases in Chinese patients screened and diagnosed by tandem mass spectrometry $[J]$. J Clin Lab Anal, 2015, 29(2): 162-168.

[16] 郑 静, 张 玉, 洪 芳, 等. 浙江省新生儿脂肪酸 氧化代谢疾病䇥查及随访分析 $[\mathrm{J}]$. 浙江大学学报 (医学版), 2017, 46(3): 248-255.

ZHENG Jing, ZHANG Yu, HONG Fang, et al. Screening for fatty acid oxidation disorders of newborns in Zhejiang province: prevalence, outcome and follow-up $[\mathrm{J}]$. Journal of Zhejiang University (Medical Sciences), 2017, 46(3): 248-255. (in Chinese)

[17] Magoulas P L, EL-HATTAB A W. Systemic primary carnitine deficiency: an overview of clinical manifestations, diagnosis, and management $[\mathrm{J}]$. Orphanet J Rare Dis, 2012, 7(1): 68.

[18 ] LEE N C, TANG N L S, CHIEN Y H, et al. Diagnoses of newborns and mothers with carnitine uptake defects through newborn screening $[\mathrm{J}]$. Mol Genet Metab, 2010, 100(1): 46-50.

[19] LI F Y, EL-HATTAB A W, BAWLE E V, et al. Molecular spectrum of SLC22A5 (OCTN2) gene mutations detected in 143 subjects evaluated for systemic carnitine deficiency[J/OL]. Hum Mutat, 2010, 31(8): E1632-E1651.

[20 ] TANG N L S, HWU W L, CHAN R T, et al. A founder mutation (R254X) of SLC22A5 (OCTN2) in Chinese primary carnitine deficiency patients $[\mathrm{J}]$. Hum Mutat, 2002, 20(3): 232.

[21］崔 冬, 胡宇慧, 唐 根, 等. 原发性肉碱缺乏症临 
床和基因突变特点及 1 例产前诊断研究 $[\mathrm{J}]$. 临床儿 科学杂志, 2019, 37(6): 449-453.

CUI Dong, HU Yuhui, TANG Gen, et al. Clinical and gene mutation characteristics of primary carnitine deficiency and prenatal diagnosis in one case $[\mathrm{J}]$. Journal of Clinical Pediatrics, 2019, 37(6): 449-453. (in Chinese)

[22] 王舒婷. 脂肪酸氧化障碍筛查与致病基因突变探 讨 [D]. 天津: 天津医科大学, 2020.

WANG Shuting. Screening and pathogenic genes analysis of fatty acid oxidation deficiencies[D]. Tianjin: Tianjin Medical University, 2020. (in Chinese)

[23] ADAM M P, ARDINGER H H, PAGON R A, et al. GeneReviews ${ }^{\circledR}[\mathrm{M} / \mathrm{OL}] / /$ EL-HATTAB A W. Systemic primary carnitine deficiency. Seattle (WA): University of Washington, 1993-2019[2020-08-01]. https://www. ncbi.nlm.nih.gov/books/NBK84551/.

[24] CHIEN Y H, LEE N C, CHAO M C, et al. Fatty acid oxidation disorders in a Chinese population in taiwan [J]. JIMD Rep, 2013, 11: 165-172.

[25] AN S J, KIM S Z, KIM G H, et al. Compound heterozygous mutations of ACADS gene in newborn with short chain acyl-CoA dehydrogenase deficiency: case report and literatures review $[\mathbf{J}]$. Korean $\mathbf{J}$ Pediatr, 2016, 59(Suppl 1): S45-S48.

[26] MINKLER P E, STOLL M S K, INGALLS S T, et al. Quantitative acylcarnitine determination by UHPLCMS/MS - going beyond tandem MS acylcarnitine “profiles" [J]. Mol Genet Metab, 2015, 116(4): 231-241.

[27] KOIZUMI A, NOZAKI J, OHURA T, et al. Genetic epidemiology of the carnitine transporter OCTN2 gene in a Japanese population and phenotypic characterization in Japanese pedigrees with primary systemic carnitine deficiency [J]. Hum Mol Genet, 1999, 8(12): 2247-2254.

[28 ] VAN MALDEGEM B T, WANDERS R J A, WIJBURG F A. Clinical aspects of short-chain acyl-CoA dehydrogenase deficiency $[\mathrm{J}]$. J Inherit Metab Dis, 2010, 33(5): 507-511.

[29] TONIN R, CACIOTTI A, FUNGHINI S, et al. Clinical relevance of short-chain acyl-CoA dehydrogenase (SCAD) deficiency: exploring the role of new variants including the first SCAD-disease-causing allele carrying a synonymous mutation $[\mathrm{J}]$. BBA Clin, 2016, 5: 114-119.

[30] PEDERSEN C B, ZOLKIPLI Z, VANG S, et al. Antioxidant dysfunction: potential risk for neurotoxicity in ethylmalonic aciduria $[\mathbf{J}]$. J Inherit Metab Dis, 2010, 33(3): 211-222.

[31] 王伟青, 李文杰, 宋东坡, 等. 短链酰基辅酶A 脱氢 酶缺乏症患儿临床特点及基因变异分析 $[\mathrm{J}]$. 临床 儿科杂志, 2020, 38(9): 687-690.

WANG Weiqing, LI Wenjie, SONG Dongpo, et al.
Clinical characteristics and gene variation of short-chain acyl-CoA dehydrogenase deficiency[J]. Journal of Clinical Pediatrics, 2020, 38(9): 687-690. (in Chinese)

[32] PENA L, ANGLE B, BURTON B, et al. Follow-up of patients with short-chain acyl-CoA dehydrogenase and isobutyryl-CoA dehydrogenase deficiencies identified through newborn screening: one center's experience[J]. Genet Med, 2012, 14(3): 342-347.

[33] OBAID A, NASHABAT M, ALFADHEL M, et al. Clinical, iochemical, and molecular features in 37 Saudi patients with very long chain acyl CoA dehydrogenase deficiency [J]. JIMD Rep, 2018, 40: 47-53.

[34] SYKUT-CEGIELSKA J, GRADOWSKA W, PIEKUTOWSKA-ABRAMCZUK D, et al. Urgent metabolic service improves survival in long-chain 3-hydroxyacylCoA dehydrogenase (LCHAD) deficiency detected by symptomatic identification and pilot newborn screening $[J]$. J Inherit Metab Dis, 2011, 34(1): 185-195.

[35 ] PENA L D M, VAN CALCAR S C, HANSEN J, et al. Outcomes and genotype-phenotype correlations in 52 individuals with VLCAD deficiency diagnosed by NBS and enrolled in the IBEM-IS database[J]. Mol Genet Metab, 2016, 118(4): 272-281.

[36] SPIEKERKOETTER U, LINDNER M, SANTER R, et al. Management and outcome in 75 individuals with long-chain fatty acid oxidation defects: results from a workshop[J]. J Inherit Metab Dis, 2009, 32(4): 488-497.

[37] BOUTRON A, ACQUAVIVA C, VIANEY-SABAN C, et al. Comprehensive cDNA study and quantitative analysis of mutant HADHA and HADHB transcripts in a French cohort of 52 patients with mitochondrial trifunctional protein deficiency $[\mathrm{J}]$. Mol Genet Metab, 2011, 103(4): 341-348.

[38 ] ROCHA H, CASTÑIRAS D, DELGADO C, et al. Birth prevalence of fatty acid beta-oxidation disorders in Iberia [J]. JIMD Rep, 2014, 16: 89-94.

[39] LOUKAS Y L, SOUMELAS G S, DOTSIKAS Y, et al. Expanded newborn screening in Greece: 30 months of experience[J]. J Inherit Metab Dis, 2010, 33(S3): 341-348.

[40 ] OERTON J, KHALID J M, BESLEY G, et al. Newborn screening for medium chain acyl-CoA dehydrogenase deficiency in England: prevalence, predictive value and test validity based on 1.5 million screened babies[J]. J Med Screen, 2011, 18(4): 173-181.

[41] MATSUBARA Y, NARISAWA K, YE-QI Y, et al. Prevalence of K329E mutation in medium-chain acylCoA dehydrogenase gene determined from Guthrie cards $[\mathrm{J}]$. Lancet, 1991, 338(8766): 552-553.

[本文编辑 沈 敏 余 方] 